\title{
Neural Networks ensemble for quality monitoring
}

\author{
P. Thomas ${ }^{1}$, M. Noyel ${ }^{1}$, M.C. Suhner ${ }^{1}$, P. Charpentier ${ }^{1}$ and A. Thomas ${ }^{1}$ \\ ${ }^{1}$ Centre de Recherche en Automatique de Nancy (CRAN-UMR 7039), Lorraine-Université, CNRS Faculté des sciences et \\ techniques, BP 70239, 54506 Vandoeuvre lès Nancy Cedex, France \\ \{philippe.thomas, melanie.noyel, marie-christine.suhner, patrick.charpentier, andre.thomas \}@univ-lorraine.fr
}

\begin{abstract}
Keywords: Neural network, product quality, neural network ensemble, Multivariate quality control, classification, classifiers ensemble.

Abstract: product quality level is a key concept for companies' competitiveness. Different tools may be used to improve quality such as the seven basic quality tools or experimental design. In addition, the need of traceability leads companies to collect and store production data. Our paper aims to show that we can ensure the required quality thanks to an "on line quality approach" based on exploitation of collected data by using neural networks tools. A neural networks ensemble is proposed to classify quality results which can be used in order to prevent defects occurrence. This approach is illustrated on an industrial lacquering process. Results of the neural networks ensemble are compared with the ones obtained with the best neural network classifier.
\end{abstract}

\section{INTRODUCTION}

One of the main goals in the mass customization context is the product quality control. Statistical process control is one of the most effective tools of Total Quality Management (TQM). The American Production and Inventory Control Society (APICS) defines Total Quality Management as "A management approach to long-term success through customer satisfaction. TQM is based on the participation of all members of an organization in improving processes, goods, services, and the culture in which they work". This definition is very related to the definition of Just in Time (JiT) because APICS presents JiT as "A philosophy of manufacturing based on planned elimination of all wastes and on continuous improvement of productivity. One of the main elements of JiT is to improve quality to zero defects. In the broad sense, it applies to all forms of manufacturing". JiT and TQM are two major concepts related to Lean manufacturing -LM- (Vollmann et al. 1984). Different tools may be used to improve quality such as the seven basic quality tools (Ishikawa chart, check sheet, control charts, histogram, Pareto chart, scatter diagram, stratification), or experimental designs. These tools, along with the Taguchi Method, are well known in the industry. Initially, quality products was controlled a posteriori and this a posteriori control led to reject or to downgrade a large part of the production. Taguchi is the first to propose to control quality before the product origination with the set-up parameters control. this approach based on the experimental design present the drawback to be off-line.

In addition, the seek of traceability imposes to companies to collect and store production data. We propose to exploit these data in order to perform online quality monitoring. The proposed approach relies to the same philosophy as Taguchi approach. The main goal is to set-up production parameters in order to prevent defects occurence. This set-up is performed on-line by taking into account the variation in the operating range.

The first step is to extrack from data, knowledge on the defects production. One important step of the knowledge discovery in data processus is the datamining step which may be performed by using neural networks. Other tools may be used to perform this step as fuzzy logic when expert knowledge is available or naïve Bayes or decision tree where only discret data are used. Neural network performs a local search of optimum. This fact implies that the neural network design needs to perform many learning with different initial weights sets in order to avoid local minimum trapping problem. These 
different learning produces different classifiers more or less performant. The simplest strategy could be to select the single, best performing classifier. Another approach consists to use an ensemble of these classifiers (Hansen and Salomon, 1990). This approach based on the Condorcet's jury theorem allows to improve the results. The second step is to performed an on-line optimal production parameter set-up based on the classifier ensemble results and which may vary in function of the operating range.

The main goal of this paper is to evaluate the use of a neural network ensemble classifier for quality monitoring process comparing with a single neural classifier. The design of a Taguchi experiments by using these classifiers is also investigated in order to find optimal tuning of controllable parameters for considered operating point.

First, we will recall succinctly the knowledge discovery in data process. In a second step, the neural network structure, its learning and pruning procedure are recalled before to present neural network ensemble. After, the industrial application case and the results obtained are presented before to conclude.

\section{KNOWLEDGE DISCOVERY IN DATA FOR QUALITY}

To control quality, we must understand it. So we need to know precisely factors affecting quality. These factors can be classified according to $6 \mathrm{M}$ (Ishikawa, 1986) of the Ishikawa chart (Machine (technology), Method (process), Material, Man Power, Measurement (inspection), Milieu (environment). When considering their controllability, only 4 of the $6 \mathrm{M}$ must be considered:

- Environmental factors (Environment-Milieu) such as temperature or humidity. These factors are generally low- or non-controllable.

- Technical factors (Machine and Method) resulting primarily from the machine state during operations. They are controllable factors.

- Human factors (Man Power) in manual operations. They are difficult to take into account because they sometimes vary consequently between operators. So far, the attempts to control human factors (establishing standards, poka yoke) have limitations and constraints.

Knowledge on the controllability of factors is needed in order to determine if a factor may be tuned or considered as constraint or even as noise. When the influential factors are correctly analyzed, we can focus on the challenge for quality control. The "zero defect" can be obtained in two ways:

- By optimizing the initial settings of various factors.

- By drifts monitoring and prevention.

This paper focuses on the first way. Historically, there are two approaches to manage quality. The first one, and also the easiest, is to use the seven basic quality tools (Ishikawa chart, check sheet, control charts, histogram, Pareto chart, scatter diagram, stratification). In this approach, the finished parts are a posteriori controlled and improvement propositions are performed by using expert knowledge. In a second approach, the main goal is to control the process and no longer the finished parts in order to tune the technical factors off-line by using experimental design methods.

In our approach, the production data collected are used in order to determine the conditions of defects apparition. To do that, a Knowledge Discovery in Data (KDD) process may be performed. KDD process is performed in different steps (Patel and Panchal, 2012). We assign a letter to each step in order to refer more easily to them later.

- Selection: obtain data from various sources (a),

- Preprocessing: cleanse data (b),

- Transformation; convert to common format, transform to new format (c),

- Data mining: obtain desired results (d),

- Interpretation/Evaluation/Presentation: present results to user in meaningful manner (e).

The two main steps are selection (a) and data mining (d). Data mining which is the core part of $\mathrm{KDD}$ is the process of analysing data and summarize it into useful information. Different approaches can be used to perform it such as artificial intelligence, machine learning, statistics and database systems. Data mining may perform different tasks:

- Classification: maps data into predefined group or classes,

- Regression: maps data from an input space to an output space,

- Clustering: groups similar data together into clusters,

- Summarization: maps data into subsets with associated simple descriptions,

- Link analysis: uncovers relationships among data.

In a quality monitoring problem, the data mining must perform a classification of data into 2 classes: defect occurrence and no defect occurrence. 


\subsection{Selection (a), preprocessing (b) and transformation (c) of data}

An important task in KDD process is the data collection (a). It is possible to collect the values of the different factors that influence quality in the same way. The availability of data is a crucial point for the quality analysis. Ideally, the collect of data must be automated. In the case of manual data collection we need to increase operator awareness of the importance of this task. Manual data collection is often seen as a waste of time because the operator must stop his work to write information not used directly in production. If this task is not correctly performed, the whole database becomes unworkable. However, manual data collection is often inevitable, because quality checking is often manually performed. It is necessary to consolidate the two types of data (production information and quality factor values). The data must be preprocessed (b) in order, for example, to synchronize the different database, delete evident outliers, and digitize qualitative data as color (c)...

\subsection{Datamining (d)}

The volume of data to be analyzed is often weighty (Agard and Kusiak 2005). Companies collect and store data for traceability reasons but they rarely use their well of information and only as indicators for real-time management methods (Kusiak, 2001). Data mining is the part of Knowledge Discovery in Data which consists in analyzing data in order to summarize it into useful information. In our case, data mining should perform a classification of data into two classes: defect occurrence and no defect occurrence. To do that, different tools may be used such as Naïve Bayes, Decision tree, Support Vector Machine (SVM), neural networks (NN)... Decision tree is faster to classify data but does not work well with noisy data (Patel and Panchal, 2012). So in the case of industrial data, the use of this approach is not pertinent. Naïve Bayes is dedicated to the treatment of discrete data and the use of continuous ones needs to perform a discretization of these data. Support Vector Machine and Neural network both use very close concepts which lead to very close results. Sometimes, SVM gives better results (Meyer et al., 2003), sometimes it is NN (Paliwal and Kumar, 2009; Hajek and Olej, 2010). This work focuses on neural network tools which are presented in the next section.

\section{NEURAL NETWORK TOOLS}

\subsection{Multilayers perceptron}

We propose the use of neural networks (NN) to model systems in order to classify quality products. The final goal is to determine the best on-line settings for each factor. The advantages of this approach are:

- Exploitation of real data without carrying out dedicated experiments as in experiments plan by using database collected during the production.

- Simple implementation of the approach because the neural model design is partially automated.

- On-line tuning of the quality monitoring process by using actual production data in order to improve and adapt the process to change.

In our approach, the neural model is performed by using production data representatives of all the conditions encountered in the past and so, it can adapt itself to these changeable conditions. This model is able to provide lower and upper limits for each controllable factor settings based on all noncontrollable factors.

The multilayer perception (MLP) seems to be the neural network best suited to our case. Works of Cybenko (1989) and Funahashi (1989) have proved that a MLP with only one hidden layer using a sigmoïdal activation function and an output layer can approximate all non-linear functions with the wanted accuracy. Its structure is given by:

$$
z=g_{2}\left(\sum_{i=1}^{n_{1}} w_{i}^{2} \cdot g_{1}\left(\sum_{h=1}^{n_{0}} w_{i h}^{1} \cdot x_{h}^{0}+b_{i}^{1}\right)+b\right)
$$

where $x_{h}^{0}$ are the $n_{0}$ inputs of the neural network, $w_{i h}^{1}$ are the weights connecting the input layer to the hidden layer, $b_{i}^{1}$ are the biases of the hidden neurons, $g_{1}($.$) is the activation function of the hidden$ neurons (here, the hyperbolic tangent), $w_{i}^{2}$ are the weights connecting the hidden neurons to the output one, $b$ is the bias of the output neuron $g_{2}($.$) is the$ activation function of the output neuron and $z$ is the network output. Because of the problem is to obtain a classification of product into two classes: defect occurrence and no defect occurrence, $g_{2}($.$) being$ chosen sigmoïdal. 


\subsection{Initialisation, learning and pruning}

Three steps must be performed in order to design the neural model.

\subsubsection{Initialisation}

The first one is the determination of the initial set of weights and biases. This step is important because learning algorithm performs a local search of minimum. So, in order to avoid local minimum trapping, different initial sets must be constructed which allow beginning learning in different zones of the criteria domain. Different initialisation algorithms have been proposed in the literature (Thomas and Bloch 1997). The initialisation algorithm used is the one proposed by Nguyen and Widrow (1990) which allows associating a random initialization of weights and biases to an optimal placement in input and output spaces. This step allows improving the diversity of the neural classifiers which is an important notion for neural network ensemble.

\subsubsection{Learning}

The second step is performed by the learning algorithm which must fit the network output with the data. In industrial application data are noisy and corrupted with many outliers. In order to limit the impact of outliers on the results, a robust LevenbergMarquardt algorithm is used (Thomas et al. 1999). Levenberg-Marquard algorithm allows associating the speed of the Hessian methods to the stability of the gradient methods. This is performed by adding a parameter multiplied by the identity matrix in order to permit the inversion of the Hessian matrix even if it is singular. The tuning of this parameter during the learning allows the Levenberg-Marquard algorithm to work as a gradient descent algorithm when this parameter is large and as a Gauss-Newton algorithm when this parameter is small. The use of a robust criterion allows avoiding the influence of outliers and, has a regularization effect in order to prevent overfitting.

\subsubsection{Pruning}

An important issue in neural network design is the determination of its structure. To determine it, two approaches can be used. The first is constructive, where the hidden neurons are added one after another (Ma and Khorasani 2004). The second approach exploits a structure with too many hidden neurons, and then prunes the least significant ones (Setiono and Leow 2000, Engelbrecht 2001). We focus on pruning approach that allows the selection simultaneously of the input neurons and the number of hidden neurons. The pruning phase is performed in two steps. First, the Engelbrecht algorithm is used which allows to quickly simplifying the structure and second the Setiono and Leow algorithm is used which is slower but also more efficient (Thomas and Thomas 2008). This step is also very important for neural networks ensemble because it allows improving performances of each classifier, and the diversity of different classifiers by differentiating there structures (pruning of inputs, hidden neurons or weights are different for the different networks).

\subsection{Neural networks ensemble}

Neural networks ensemble is an interesting approach to improve quality of classifier. Typically, an ensemble classifier can be built at four different levels (Kuncheva 2004): Data level (Breiman 1996), feature level (Ho, 1998), classifier level and combination level (Kuncheva 2002). We focus here on classifier and combination level. The principle of neural network ensemble is presented figure 1 . Design of ensemble neural networks consists of two main steps: the generation of multiples classifiers and their fusion (Dai 2013). This leads to two problems: how many classifiers are needed and how to perform their fusion.

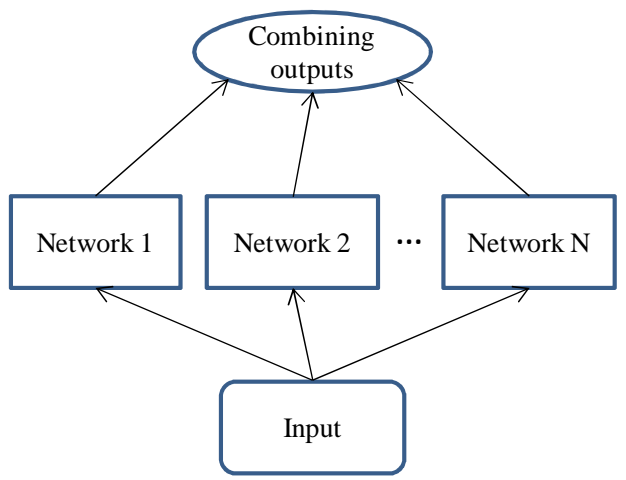

Figure 1: A neural network ensemble.

The classifier selection is a problem addressed by many authors (Ruta and Gabris 2005, HernandezLobato 2013, Dai 2013). Two approaches may be used to perform it (Ruta and Gabrys 2005):

- Static classifiers selection. The optimal selection solution found for the validation set 
is fixed and used for the classification of unseen patterns.

- Dynamic classifiers selection. The selection is done on-line, during classification, based on training performances and also various parameters of the actual unlabelled pattern to classify.

In order to preserve speed of classification, we use here a static classifiers selection. Different selection criterions have been proposed. Individual best performance is an universal indicator for selection of the individual best classifiers, which is the simplest and is reliable and robust is generally preferred in industrial applications (Ruta and Gabrys 2005). This indicator calls minimum individual error (MIE) represents the minimum error rate of the individual classifier and promotes individual best classifiers selection strategy:

$$
M I E=\min _{j}\left(\frac{1}{n} \sum_{i=1}^{n} e_{j}(i)\right)
$$

where $e_{j}(i)$ represents the error classification of classifier $j$ on data $i$.

We use this criterion to select 100 classifiers in order to benefit of a large classifiers set and to obtain directly a percentage of vote representative to the confidence of classification. Ensemble pruning algorithm may also be used in order to optimize the size of the classifiers ensemble (Tsoumakas et al. 2009, Guo and Boukir 2013).

The fusion of classifiers is generally performed by majority vote. Kuncheva et al. (2003) have proposed to implement limits on the majority vote accuracy in classifier fusion. They studied the problem of lack of independence in the classifiers which may limit the interest of classifiers ensemble. We implement majority vote which presents the advantage that it could be also used as a confidence interval on the classification results. This approach is applied on an industrial quality control.

\section{INDUSTRIAL APPLICATION}

\subsection{Presentation of the process}

The considered company produces high quality lacquered panels made in MDF (Medium Density Fiberboard) for kitchens, bathrooms, offices, stands, shops, hotel furniture. Its main process is a robotic lacquering workstation. Even if this workstation is free of human factors, the production quality is unpredictable (we cannot know if there is a risk that products will have defects) and fluctuates (the percentage of defects may be of $45 \%$ one day and down to $10 \%$ the next day without changing the settings). After a brainstorming about the factors influencing the quality level, we were able to classify them in three categories specified in paragraph 2. We could imagine the potential drifts and associate a type of defect. This preliminary work results of expert knowledge. Only after the complete study can we know accurately which factors affect which defects. Thanks to a production and quality management system, data corresponding to the factors studied since february 2012 to september 2012 have been collected. Upstream of the robotic lacquering workstation, experts decided to collect factors such as load factor, number of passes, time per table (lacquering batches), liter per table, basis weight, number of layers, number of products and drying time. We could add to these technical factors environmental ones such as temperature, atmospheric pressure and humidity. According to the experimental design approach, we can classify these variables into two types of factors: internal ones (load factor, number of passes, time per table, liter per table, basis weight, number of layers, number of products and drying time) and external ones (temperature, humidity, pressure). Two factors (passes number and number of layers) are discrete ones that can each take three states and that are binarized (Thomas and Thomas 2009).

We have 15 inputs to apply to the neural network which 6 are binary ones. Downstream of the machine, we were able to detect up to 30 different types of defects. The first works with the neural network prediction concerning a type of defects: "Stains on back." We have a total of 2270 data we will split into 2 data sets for identification (1202 data) and validation (1068 data). First, learning is achieved by exploiting 25 neurons in the hidden layer. Pruning phase can then eliminate spurious inputs and hidden neurons. This is done 150 times with different initial weights sets to avoid problem of trapping in local optimum. The 100 better classifiers are used in the neural networks ensemble and the performances of the neural networks ensemble are compared with the best neural classifier. The different initialisation sets and the pruning phase allows to assure diversity into the classifers set.

During the validation phase, we therefore compare the results of the best neural classifier with the real defects detection. For the defect "Stains on back", we know that it occurs 127 times on the 1068 data validation set. The best neural classifier can 
detect 112 defects which lead to a non-detection rate of $11.8 \%$. The proportion of false positive is $19.2 \%$, which may be partly explained by the fact that some defects haven't been identified out of the machine. The quality control is performed manually. This fact induces that some defects are not notice.

The using of neural networks ensemble on the same validation data set gives a non-detection rate of $10.2 \%$. The proportion of false positive is $16.4 \%$. These results show an improvement of $14 \%$ of the non-detection rate and $15 \%$ on the false alarme rate. The same work performed on a second defect (grain on face, 477 defects on the 1068 data validation set) allows to reduce the non detection rate from $42 \%$ to $32 \%$ ( $24 \%$ of improvement) and a false alarme rate from $21 \%$ to $16 \%$ ( $24 \%$ of improvement).

Another advantage of the neural networks ensemble is that the result of the vote may be use as a confidence interval on the classification result. As example, for a data, a vote with $40 \%$ of defect and $60 \%$ on non defect may induces to suspect the occurence of defect even if the classification result leads to a non defect classification.

It is obvious that these defects are largely explained by the archived operating conditions and it's possible to use neural networks ensemble upstream of the workstation to prevent the risk of defects. However, there are defects which cannot be predicted with the neural networks ensemble in the conditions described above. This is for example the case for "knock" where you get $73 \%$ non-detection for $11 \%$ false positives. The non predictable defects depend certainly on other factors that we need to determine and collect if we want to predict them. In total, on the 30 identified defects, 7 can be partially explained using the variables collected. For the predictable defects, there are 2 possible approaches:

- Warning. By analyzing the inputs through the neural networks ensemble, it becomes possible to predict defects occurrence and report it when conditions are met to create risk.

- Limitation. By using neural networks ensemble to limit the input factors by upper and lower limits and prohibit the production lot when one of the inputs is outside these limits. If it's a controllable factor, operators can modify it to allow production. Otherwise, the production lot will be rejected. It must be scheduled later when conditions become acceptable. To do that, factors must be classified into controllable ones (load factor, basis weight, drying time, liters per table), non-controllable ones (temperature, humidity, pressure) and protocols (number of passes, time per table, number of layers, and number of products). The neural networks ensemble is then used instead of the real system to perform experiments that achieve an entire plan without increasing the cost. The results, however, still to be validated on the real system.

Figure 2 and 3 present the results of an entire Taguchi plan in which 10 levels were chosen for the 3 controllable factors. In this example, for protocol factors, we set the number of passes and layer to 1 ; the time per table, and the liter of lack to their average values; and the number of products to its median value. We also fixed non-controllable factors values to their average values. The effect of each factor $x_{i}$ to a level $A_{i}$ is given classically by the mean of all the results (defect occurence) obtained when $x_{i}=A_{i}$ minus the mean of the results obtained with all the experiences. When an effect is positive, this implies that the considered level increase the occurence of defects, when it is negative, the considered level reduce the occurence of defects. The interractions between factor may be investigated in the same way.

Figure 2 presents the results obtained with the best neural classifier. It shows that the load factor has a relatively small impact on the defects occurrence. However, the increase in weight has a significant effect. High basis weights tend to create more defects. Drying time has also an impact because too short drying time greatly increases the occurrence of defects.

Figure 3 presents the same work performed by using neural networks ensemble. Due to the diversity of the different classifiers, the impact of each effect are presented in the forme of an enveloppe including the effects for all the classifiers. We can see that the information given by the neural networks ensemble is more complete than the one given by the best neural classifier. If, for the load effect, the neural networks ensemble confirms that it has a very small impact on the defects occurence, for the basis weight effect and dry effect, it shows that small and large values may lead to defects occurence.

This information can not be obtained by using uniquely the best neural classifier. These results are useful to tune the process parameter to their best values under the constraint of uncontrollable factors as meteorological ones. 

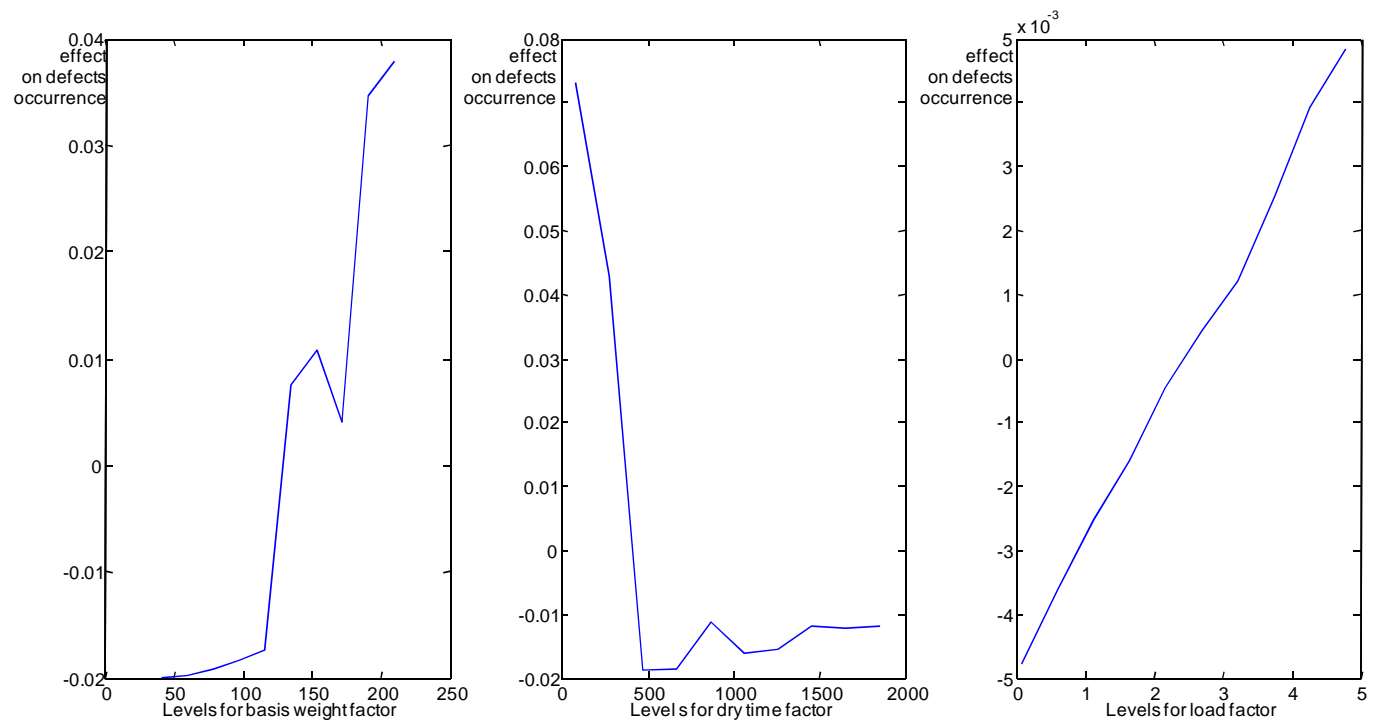

Figure 2: Experiment plan results by using best NN classifier.
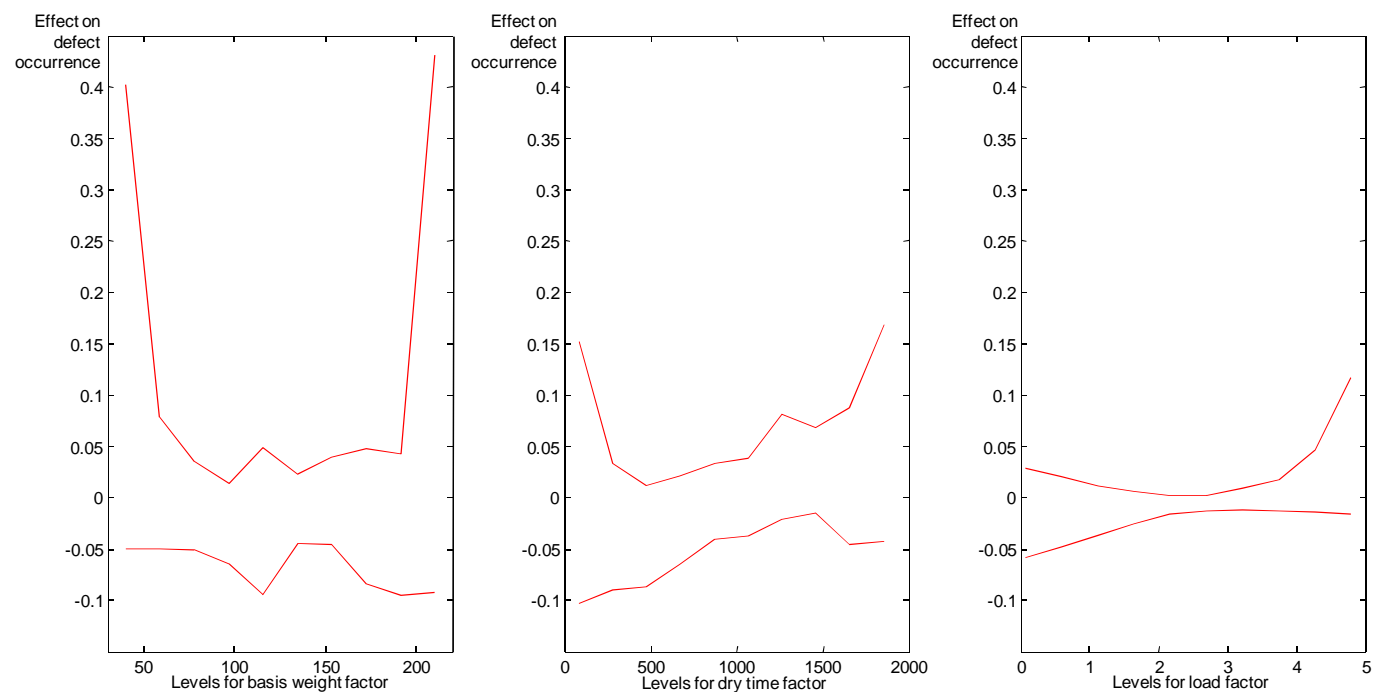

Figure 3: Experiment plan results by using neural networks ensemble.

As example, with the operating range considered here, the set-up of the basis weight may be tuned between 90 and 190 when the tuning of dry time may be tuned between 400 and 1100 in order to limit the risk of defects occurence.In order to obtain the same results by using an experiments plan, we need to use 5 modalities for drying time and basis weight and 2 for the load factor that lead to many experiments even using Taguchi plan. These preliminary results need to be confirmed by taking into account the variation of non-controllable factors (temperature, pressure and humidity) and validating the results on the real system.

\section{CONCLUSIONS}

This paper presents neural networks ensemble for quality monitoring comparing to single neural classifier. The approach is applied and tested on an industrial application. The results show that a neural networks ensemble allows improving greatly the classification performance. We show that neural networks ensemble, as single classifier, allows performing Taguchi experiments in order to find the best tuning of parameters in order to avoid defects 
occurrence. Due to diversity of classifier, the results of Taguchi experiments obtained by using neural networks ensemble are more complete and useful.

Two ways of improvement must be performed in the future works. The first one is to use pruning ensemble algorithm in order to optimize the size of the ensemble in function of the results. The second one is to improve the diversity of classifiers by using other classifiers tools as support vector machines, fuzzy logic or genetic algorithms.

\section{REFERENCES}

Agard, B., Kusiak, A., 2005. Exploration des bases de données industrielles à l'aide du datamining Perspectives. $9^{\text {ème }}$ colloque national AIP PRIMECA.

Breiman, L., 1996. Bagging predictors. Machine Learning, 24, 2, 123-140.

Cybenko, G., 1989. Approximation by superposition of a sigmoïdal function. Math. Control Systems Signals, 2, 4, 303-314.

Dai, Q., 2013. A competitive ensemble pruning approach based on cross-validation technique. KnowledgeBased Systems. 37, 394-414.

Engelbrecht, A.P., 2001. A new pruning heurisitc based on variance analysis of sensitivity information. IEEE trasanctions on Neural Networks, 1386-1399.

Funahashi, K., 1989. On the approximate realisation of continuous mapping by neural networks. Neural Networks, 2, 183-192.

Guo, L., Boukir, S., 2013. Margin-based ordered aggregation for ensemble pruning. Pattern Recognition Letters, 34, 603-609.

Hajek, P., Olej, V., 2010. Municipal revenue prediction by ensembles of neural networks and support vector machines. WSEAS Transactions on Computers, 9, 1255-1264.

Hansen, L.K., Salomon, P., 1990. Neural network ensembles. IEEE Transactions on Pattern Analysis and Machine Intelligence. 12, 10, 993-1001.

Hernandez-Lobato, D., Martinez-Munoz, G., Suarez, A., 2013. How large should ensembles of classifiers be? Pattern Recognition. 46, 1323-1336.

Ho, T., 1998. The random subspace method for constructing decision forests. IEEE Transactions on Pattern Analysis and Machine Intelligence. 20, 8, 832844.

Ishikawa, K., 1986. Guide to quality control. Asian Productivity Organization.

Kuncheva, L.I., 2002. Switching between selection and fusion in combining classifiers: An experiment. IEEE Transactions on Systems, Man and Cybernetics, part B: Cybernetics. 32, 2, 146-156.

Kuncheva, L.I., 2004. Combining pattern classifiers: Methods and algorithms. Wiley-Intersciences.
Kuncheva, L.I., Whitaker, C.J., Shipp, C.A., 2003. Limits on the majority vote accuracy in classifier fusion. Pattern Analysis and Applications. 6, 22-31.

Kusiak, A., 2001. Rough set theory: a data mining tool for semiconductor manufacturing. Electronics Packaging Manufacturing, IEEE Transactions on, 24, 44-50.

Ma, L., Khorasani, K., 2004. New training strategies for constructive neural networks with application to regression problems. Neural Network, 589-609.

Meyer, D., Leisch, F., Hornik, K., 2003. The support vector machine under test. Neurocomputing, 55, 169186.

Nguyen, D., Widrow, B., 1990. Improving the learning speed of 2-layer neural networks by choosing initial values of the adaptative weights. Proc. of the Int. Joint Conference on Neural Networks IJCNN'90, 3, 21-26.

Paliwal, M., Kumar, U.A., 2009. Neural networks and statistical techniques: A review of applications. Expert Systems with Applications, 36, 2-17.

Patel, M.C., Panchal, M., 2012. A review on ensemble of diverse artificial neural networks. Int. J. of Advanced Research in Computer Engineering and Technology, 1, 10, 63-70.

Ruta, D., Gabrys, B., 2005. Classifier selection for majority voting. Information Fusion. 6, 63-81.

Setiono, R., Leow, W.K., 2000. Pruned neural networks for regression. 6th Pacific RIM Int. Conf. on Artificial Intelligence PRICAI'O0, Melbourne, Australia, 500509.

Thomas, P., Bloch, G., 1997. Initialization of one hidden layer feedforward neural networks for non-linear system identification. $15^{\text {th }}$ IMACS World Congress on Scientific Computation, Modelling and Applied Mathematics WC'97, 4, 295-300.

Thomas, P., Bloch, G., Sirou, F., Eustache, V., 1999. Neural modeling of an induction furnace using robust learning criteria. J. of Integrated Computer Aided Engineering, 6, 1, 5-23.

Thomas, P., Thomas, A., 2008. Elagage d'un perceptron multicouches : utilisation de l'analyse de la variance de la sensibilité des paramètres. $5^{\text {ème }}$ Conférence Internationale Francophone d'Automatique CIFA'08. Bucarest, Roumanie.

Thomas, P., Thomas, A., 2009. How deals with discrete data for the reduction of simulation models using neural network. $13^{\text {th }}$ IFAC Symp. On Information Control Problems in Manufacturing INCOM'09, Moscow, Russia, june3-5, 1177-1182.

Tsoumakas, G., Patalas, I., Vlahavas, I., 2009. An ensemble pruning primer. in Applications of supervised and unsupervised ensemble methods $O$. Okun, G. Valentini Ed. Studies in Computational Intelligence, Springer.

Vollmann, T.E., Berry, W.L. and Whybark, C.D., 1984. Manufacturing Planning and Control Systems, Dow Jones-Irwin. 